\section{Genetische Grundlagen für die Therapie chronisch entzündlicher Barriereerkrankungen}

\author{
S. Schreiber ${ }^{1}$ \\ 1 Direktor der Klinik für Innere Medizin I und Direktor des Instituts \\ für Klinische Molekularbiologie, Christian-Albrechts-Universität, \\ Universitätsklinikum Schleswig-Holstein
}

Die Häufung von Erkrankungsfällen in großen Familien, aber auch die Beobachtung, dass in eineiigen Zwillingspaaren eine erhöhte Konkordanz im Vergleich zu zweieiigen Zwillingen auftritt, suggeriert eine genetische Veranlagungskomponente für chronisch entzündliche Erkrankungen der Barriereorgane. Interessanterweise finden sich familiäre Häufungen über mehrere Krankheitsentitäten hinweg (z. B. gemischte Familien mit Morbus Crohn und Colitis ulcerosa oder Morbus Crohn und Psoriasis). In einigen der chronisch entzündlichen Barriereerkrankungen weist eine sehr hohe Konkordanz (z. B. bei Morbus Crohn: monozygote Zwillinge $-55 \%$ vs. dizygote Zwillinge -5\%) auf eine sehr starke genetische Komponente im Krankheitsrisiko hin. Keine der chronisch entzündlichen Barriereerkrankungen folgt jedoch einem Mendel'schen Erbgang. Es handelt sich um typische komplexe Erkrankungen, die mit hoher Wahrscheinlichkeit eine große Zahl von interagierenden Krankheitsgenen und -varianten involvieren und zudem noch durch Umweltfaktoren in suszeptiblen Individuen ausgelöst werden.

Der Morbus Crohn ist ein paradigmatisches Beispiel für die erfolgreiche Exploration einer polygenen Ätiologie geworden. Morbus Crohn war vor 1920 nicht bekannt gewesen, bis die ersten Fälle in Schottland und in USA beschrieben wurden. Die Inzidenz hat sich seitdem stetig erhöht. Derzeit wird von einer Lebenszeitprävalenz von bis zu $0,5 \%$ in nordwestlichen Hochrisikopopulationen ausgegangen. Die derzeitige Hypothese ist, dass es unklare Faktoren im Lebensstil der westlichen Industriegesellschaften gibt, die mit einer polygenen Suszeptibilität interagieren und dadurch zur Krankheitsmanifestation führen.

Pathophysiologisch haben chronisch entzündliche Barriereerkrankungen gemeinsam, dass die Produktion von TNF und anderen proentzündlichen Zytogenen erhöht ist und es gleichzeitig auch zu einer Aktivierung des NF-KB-Systems kommt. Die Pathophysiologie ist jedoch heterogen und nur auf der Effektorebene auflösbar. Die Analyse der Pathophysiologie konnte jedoch nicht zu einer Differenzierung des Gesamtkrankheitsbilds in Patientenuntergruppen führen. Auch der Versuch, klinische Einteilungen als Leitfaden für die Entdeckung einer differenziellen Pathophysiologie zu machen, ist gescheitert. Selbst der Einsatz moderner genomischer Techniken, wie cDNA oder oligonukleotidbasierte Mikroarrays hat zwar zu einem weiteren Aufbruch der pathophysiologischen Signalkaskade geführt, jedoch nicht die Ursachen der Erkrankung definiert oder die Patienten in verschiedene Erkrankungstypen kategorisiert.
2010 sind in der Analyse der genetischen Ätiologie 3 verschiedene kodierende Varianten im NOD2-Gen identifiziert worden, die statistisch in hohem Maße mit der Erkrankung assoziiert sind. Alle 3 Varianten betreffen einen Teil des Gens, der für den leucinreichen Repeat-Terminus des Proteins kodiert und die Sensordomäne für Muramyl-Dipeptid ist. Dadurch kommt es zur Aktivierung von NF-KB in Makrophagen und Epithelzellen. Es konnte auch ein statistischer Zusammenhang mit der anatomischen Lokalisation der Krankheit in der Ileozökalregion hergestellt werden. Die hier entdeckten Varianten im NOD2-Gen sind interessanterweise auch an anderen entzündlichen Erkrankungen beteiligt. So konnte in der Folge ein Zusammenhang auch zu Asthma bronchiale, psoriatischer Arthritis, Periodontitis und dem kolorektalen Karzinom hergestellt werden. Diese Zusammenhänge sind jedoch statistisch deutlich schwächer als die Assoziation zu Morbus Crohn.

In der weiteren genetischen Exploration der Ursachen des Morbus Crohns zeigte sich dann, dass die Varianten im NOD2-Gen bei Weitem nicht das genetische Risiko erklärten. Durch die Verfügbarkeit von hochdichten SNP-Arrays, die bis zu 1 Million Einzelbasenpolymorphismen pro Individuum abfragen, war es möglich, in sehr großen Kohorten von Morbus-Crohn-Patienten und Kontrollen eine Vielzahl von weiteren Krankheitsgenen zu entdecken. In großen Metaanalysen und auch durch den Einsatz eines spezifischen, auf Genvarianten in immunrelevanten Genen fokussierten Chips sind derzeit mehr als 160 Krankheitsgene für chronisch entzündliche Darmerkrankungen identifiziert worden. Trotzdem ist das genetische Risiko dadurch noch nicht vollständig beschrieben. In der Analyse der medizinischen Systembiologie der Erkrankung zeichnet sich doch eine Reihe von unterschiedlichen Stoffwechselwegen ab, die bei der Erkrankung beteiligt zu sein scheinen. Hierzu gehören die angeborene Immunität, Zytokinregulation, insbesondere im Bereich von IL23/IL12 und STAT3, die Autophagie und endosomale Stressreaktionen.

Parallel zum Morbus Crohn sind genomweite Assoziationsstudien (GWAS) auch bei Colitis ulcerosa und anderen entzündlichen Barriereerkrankungen durchgeführt worden. Wie bei Morbus Crohn zeigt sich eine Vielzahl assoziierter Krankheitsgene und Varianten. Häufig stellen die entdeckten Varianten auch nur Marker für ein genetisches Signal dar, das sich dann durch konsequente Sequenzierung des Locus in eine Vielzahl seltener, z.B. kodierender Polymorphismen umsetzen lässt. Interessanterweise zeigt die Analyse anderer entzündlicher Barriereerkrankungen eine erhebliche Überlappung der beteiligten Krankheitsgene zwischen den diagnostischen Entitäten. Hierdurch sind klinische Beobachtungen erklärbar, dass oft die Phänotypen zwischen Morbus Crohn und Colitis ulcerosa zu wechseln scheinen. Wahrscheinlich ist in vielen Patienten die polygene Suszeptibilität auch nicht in dem Maße abgrenzbar, wie sich das der Kliniker für seine Diagnostik wünschen würde. Im Gegenteil, es steht zu erwarten, dass die diagnostische Einteilung, die vorwiegend organbezogen definiert wurde, möglicherweise in $\mathrm{Zu}-$ kunft revidiert werden muss und dass auch primäre organübergreifende Manifestationen im Krankheitsverlauf deutlich zu wenig klinisch beachtet wurden. 
Eine der wesentlichen Erkenntnisse des Verständnisses der Systembiologie der Erkrankungen durch die breite genetische Exploration ist, dass es eine einheitliche Hypothese zur Pathophysiologie nicht gibt. Weiterhin sind eine Reihe pathophysiologischer Therapieansätze (z.B. alleinige Fokussierung auf T-Zell-Aktivierung) auch schon aus genetischer Sicht nicht erfolgreich. Dies wird auch durch die klinische Beobachtung belegt, dass bislang keines der fokussierten Therapieverfahren in der Behandlung dieser Erkrankungen in mehr als $30-40 \%$ der Patienten eine positive Therapieantwort zeigt. Die weitere genetische Exploration der chronisch entzündlichen Barriereerkrankungen wird in einer Vervollständigung der genetischen Risikokarten resultieren. Es ist zu erwarten, dass dieses angesichts der Verfügbarkeit von Höchstdurchsatz-Sequenziertechnologien mit erheblicher Geschwindigkeit vorangeht. Die Vervollständigung der systembiologischen Modelle der Erkrankungen wird sicherlich auch zur Etablierung und Nutzung neuer Therapieverfahren führen. Es ist zu erwarten, dass solche therapeutischen Ansätze im Blick der bislang verwandten Diagnosen krankheitsübergrei- fend konzipiert werden müssen. Ähnlich der Entwicklung für gezielte Therapieansätze in der Onkologie scheint es wichtig zu sein, Kategorien von Patienten zu bilden, die jenseits des organbezogenen Indikationsbegriffs definiert werden. Zukünftige Therapien, die z.B. spezifische Defekte in der angeborenen Immunität adressieren, müssen daher vorwiegend an Patienten getestet werden, die diesen Defekt auch aufweisen, unabhängig davon, ob es zu einer entzündlichen Manifestation im Bereich ihrer Schleimhäute oder einer bestimmten Krankheitskategorie kommt.

Interessenkonflikt: Keiner

\section{Bibliografie}

DOI http://dx.doi.org/10.1055/s-0032-1324913

Arzneimittelforschung 2012; 62, Suppl. 1: S20-S21

(c) Georg Thieme Verlag KG Stuttgart · New York .

ISSN 0004-4172 